\title{
$\theta$-REGULAR SPACES
}

\section{DRAGAN S. JANKOVIĆ}

\author{
Department of Mathematics \\ Texas Tech University \\ Lubbock, Texas 79409 U.S.A. \\ (Received May 29, 1984)
}

$\Lambda$ BSTRACT. In this paper we define a topological space $X$ to be $\theta$-regular if every filterbase in $X$ with a nonempty v-adherence has a nonempty adherence. It is shown that the class of $u$-regular topological spaces includes rim-compact topological spaces and that $H$-regular $H(i)$ (Hausdorff) topological spaces are compact (regular). The concept of 0 -regularity is used to extend a closed graph theorem of Rose [1]. It is established that an $r$-subcontinuous closed graph function into a $\theta$-regular topological space is continuous. Another sufficient condition for continuity of functions due to Rose [1] is also extended by introducing the concept of almost weak continuity which is weaker than both weak continuity of Levine and almost continuity of Husain. It is shown that an almost weakly continuous closed graph function into a strongly locally compact topological space is continuous.

KEY WORDS AND PHRASES. O-regular spaces, rim-compact spaces, closed graph functions. 1980 'LATHCMATICS SUBJECT CLASSIFICATION CODE. Primary 54C10; Secondary 54C05, 54D45.

\section{INTRODUCTION}

In a recent paper, Rose [1] presented a strong version of Levine's decomposition theorem and applied it to show that a closed graph weakly continuous function into a rim-compact topological space is continuous and that a closed graph almost continuous function into a strongly locally compact topological space is continuous. One of the purposes of the present note is to extend these results. In Section 3 we introduce the class of 0 -regular topological spaces containing both the class of regular spaces and the class of rim-compact spaces and show that $A$-regular $H(i)$ ( $R_{1}$ ) topological spaces are compact (regular). In Section 4 it is established that a closed graph $r$-subcontinuous function into a ${ }^{-}$-regular topological space is continuous. As a consequence we have that a closed graph weakly continuous function into a $\theta-r e g u l a r$ topological space is continuous. The new weak form of continuity, called almost weak continuity, which is weaker than both weak continuity and almost weak continuity, is introduced and it is obtained that an almost weakly continuous C-continuous function into a strongly locally compact topological space is continuous. As a consequence we have that a closed graph almost weakly continuous function into a strongly locally compact topological space is continuous.

2. PRELIMINARIES.

Throughout, spaces mean topological spaces and the closure, interior and boundary 
of a subset $A$ of a space are denoted by $\mathrm{Cl}(\mathrm{A})$, Int $(\mathrm{A})$ and $\mathrm{Fr}(\mathrm{A})$, respectively. A point $x$ of a space $X$ is in the $\theta$-closure of a subset $A$ of $X\left(x \in C 1_{\theta}(A)\right.$ ) if each closed neighborhood $U$ of $x$ satisfies $U \cap A \neq \emptyset$ (Veličko [2]). The $\theta$-adherence of a filterbase $\nabla\left(\operatorname{ad}_{\theta} \nabla\right)$ in a space $X$ is $\cap\left\{C 1_{\theta}(F): F \varepsilon \nabla\right\}$ and $\nabla \theta$-convergence to $x$ if for each open set $U$ containing $x$ there is an $F \in V$ such that $F \subset C 1(U)$ (Veličko [2]). It is clear that in regular spaces b-adherence of a filterbase coincides with the adherence. A space $X$ is said to be $H(i)$ (Scarborough and Stone [3]) if every open covering of $X$ has a Iinite subcollection whose closures cover X. From the characterization of H-closed spaces due to Veličko [2] it can be seen that a space $X$ is $H(i)$ if and only if $\left.\operatorname{ad}^{\prime}\right\rangle \neq$ for each filterbase $\nabla$ in $X$. A space is called rim-compact if each $x$. $X$ has a local baise of open sets with compact boundaries. A space is said to be strongly locally compact if each $x \in X$ has a closed compact neighborhood. Every locally compact regular spice is strongly locally compact and every strongly locally compact space is rimcompact. There exist strongly locally compact spaces which are not regular (Example 73 due to Steen and Seebach [4]). We employ as a primitive the following characteriz.1tion of weak continuity (Levine [5]) (resp. almost continuity (Husain [6])) from (Levine [5]) (resp. (Rose [1])). A function $\mathrm{f}: \mathrm{X} \rightarrow \mathrm{Y}$ is weakly continuous (resp. almost continuous) if $\mathrm{f}^{-1}(\mathrm{~V}) \subset \operatorname{Int}\left(\mathrm{f}^{-1}(\mathrm{Cl}(\mathrm{V}))\right)\left(\operatorname{resp} \cdot \mathrm{f}^{-1}(\mathrm{~V}) \subset \operatorname{Int}\left(\mathrm{Cl}\left(\mathrm{f}^{-1}(\mathrm{~V})\right)\right)\right)$ for each open subset $V$ of $Y$. Weak continuity and almost continuity are independent conditions (Rose [7]).

3. (1-REGULAR SPACES.

DEFINITION 3.1. A space $X$ is 0 -regular if every filterbase in $X$ with a nonempty 1 -, ldherence has a nonempty adherence.

It is clear that regular spaces are $\theta$-regular. The following theorem shows that an important class of spaces is also contained in the class of 9 -regular spaces.

THEOREM 3.2. Rim-compact spaces are 0-regular.

PROOF. Let $X$ be a rim-compact space and let $V$ be a filterbase in $X$ such that ad, $V \neq \emptyset$. Assume that $\operatorname{ad} \nabla=\emptyset$ and let $x \varepsilon \operatorname{ad}_{\theta} \nabla$. Then there exist an open set $V$ containing $x$ and an $F_{1} \varepsilon \nabla$ such that $V \cap F_{1}=\emptyset$. Since $X$ is rim-compact, there exists an open set $U$ containing $x$ such that $U \subset V$ and $\operatorname{Fr}(U)$ is compact. Suppose that $\operatorname{Fr}(U)=\emptyset$. Then $\mathrm{Cl}(\mathrm{U})=U$ and since $\mathrm{x}<\operatorname{ad}_{\theta} \nabla, U \cap \mathrm{F}_{1} \neq \emptyset$. This is a contradiction and hence $\operatorname{Fr}(U) \neq \emptyset$. We show that $\operatorname{Fr}(U) \cap F \neq \emptyset$ for each $F \& \nabla$. To do so assume that there is an $\mathrm{F}_{2}, 7$ such that $\operatorname{Fr}(\mathrm{U}) \cap \mathrm{F}_{2}=\emptyset$. Choose $\mathrm{F} \& \nabla$ such that $\mathrm{F} \subset \mathrm{F}_{1} \cap \mathrm{F}_{2}$. Then $\mathrm{U} \cap \mathrm{F}=\emptyset$ and $\operatorname{Fr}(U) \cap F=\emptyset$ so that $\mathrm{Cl}(\mathrm{U}) \cap \mathrm{F}=\emptyset$. This contradicts the assumption that $\mathbf{x} \varepsilon \operatorname{ad}_{\hat{\theta}} \nabla$. We conclude that $\operatorname{Fr}(U) \cap F \neq \emptyset$ for each $F \& \nabla$. Since $\operatorname{Fr}(U)$ is compact, $\operatorname{Fr}(U) \cap \operatorname{adV} \neq \emptyset$. This contradiction establishes the proof.

It is well known that regular $\mathrm{H}(\mathrm{i})$ spaces are compact. The corollary to the following readily established result reveals that rim-compact $H(i)$ spaces are also compact .

THEOREM 3.3. An H(i) space is compact if and only if it is $\theta$-regular.

COROLLARY 3.4. An H(i) space is compact if and only if it is rim-compact.

The concept of $R_{1}$ spaces was introduced by Davis [8]: A space $X$ is $R_{1}$ if for each $\mathrm{x}, \mathrm{y} \varepsilon \mathrm{X}$ with $\mathrm{Cl}\{\mathrm{x}\} \neq \mathrm{C} 1\{\mathrm{y}\}$ there exist open disjoint sets $\mathrm{U}$ and $\mathrm{V}$ such that $\mathrm{C} 1\{\mathrm{x}\} \subset \mathrm{U}$ and $\mathrm{Cl}\left\{\mathrm{y} ; \subset \mathrm{V}\right.$. He showed that the class of $\mathrm{R}_{1}$ spaces contains both the class of 
regular spaces and the class of Hausdorff spaces. Janković [9] established that a space $X$ is $R_{1}$ if and only if $C l_{\theta}\{x\}=C 1\{x\}$ for each $x \varepsilon X$,

LEMMA 3.5. A space $X$ is $R_{1}$ if and only if $\operatorname{ad} \nabla \subset C 1\{x\}$ for each filterbase $\nabla$ $\theta$-converging to $\mathrm{x} \varepsilon \mathrm{X}$.

PROOF. Let $\nabla$ be a filterbase in an $R_{1}$ space $x \theta$-converging to $x \in x$. If $\operatorname{ad} \nabla=\emptyset$ we are done. Suppose that $\operatorname{ad} \nabla \neq \emptyset$. Let $y \varepsilon$ ad $\nabla$ and $y \notin C 1_{\theta}\{x\}$. Then there exists an open set $U$ containing $y$ such that $x \notin C l(U)$. Since $\nabla \theta$-converges to $x$ there exists an $\mathrm{F} \varepsilon \nabla$ such that $\mathrm{F} \subset \mathrm{Cl}(\mathrm{X}-\mathrm{Cl}(\mathrm{U}))$. This implies that $\mathrm{F} \cap \mathrm{U}=\emptyset$ which contradicts the assumption that $y \varepsilon \operatorname{ad} \nabla$. We conclude that $y \in C 1_{\theta}\{x\}$ and $\operatorname{ad} \nabla \subset C 1_{\theta}\{x\}$. Since $X$ is $R_{1}$, $\mathrm{C} 1_{\theta}\{\mathrm{x}\}=\mathrm{Cl}\{\mathrm{x}\}$ and hence $\operatorname{ad} \nabla \subset \mathrm{Cl}\{\mathrm{x}\}$.

Conversely, let $x \in X$. It is obvious that the filterbase $\nabla$ of all open sets containing $x \theta$-converges to $x$. Since $\operatorname{ad} \nabla=\cap\{C 1(U): U$ is open and $x \in U\}=C 1_{\theta}\{x\}$, by hypothesis it follows that $\mathrm{Cl}_{\theta}\{\mathrm{x}\} \subset \mathrm{Cl}\{\mathrm{x}\}$. This shows that $\mathrm{X}$ is $\mathrm{R}_{1}$.

THEOREM 3.6. Every $\theta$-regular $R_{1}$ space is regular.

PROOF. Let $A$ be a subset of a $\theta$-regular $R_{1}$ space $X$ and let $x \in C 1_{\theta}(A)$. Then $\mathrm{Cl}(\mathrm{U}) \cap \mathrm{A} \neq \emptyset$ for each open set $\mathrm{U}$ containing $\mathrm{x}$ and $\nabla=\{\mathrm{Cl}(\mathrm{U}) \cap \mathrm{A}$ : $\mathrm{U}$ is open and $\mathrm{x} t \mathrm{U}\}$ is a filterbase $\theta$-converging to $x$. Since $X$ is $\theta$-regular, there exists a y $\varepsilon$ ad $\nabla$. From Lemma 3.5 it follows that $y \in C 1\{x\}$ and consequently, $x \in C 1\{y\}$ since $x$ is $R_{1}$. But $C 1\{y\} \subset \operatorname{ad} \nabla \subset \mathrm{Cl}(\mathrm{A})$ so that $\mathrm{x} \in \mathrm{Cl}(\mathrm{A})$. Therefore $\mathrm{Cl}_{\theta}(\mathrm{A}) \subset \mathrm{Cl}(\mathrm{A})$. This shows that $\mathrm{X}$ is regular.

COROLLARY 3.7. Every $\theta$-regular Hausdorff space is regular.

4. SUFFICIENT CONDITIONS FOR CONTINUITY.

The concept of a subcontinuous function, which is a generalization of a function whose range is compact, was introduced by Fuller [10]: A function $\mathrm{f}: \mathrm{X} \rightarrow \mathrm{Y}$ is said to be subcontinuous if ad $f(\nabla) \neq \emptyset$ for each convergent filterbase $\nabla$ in $X$. Recently, Herrington [11] defined a function $f: X \rightarrow Y$ to be $r$-subcontinuous if $\operatorname{ad}_{\theta} f(\nabla) \neq \emptyset$ for each convergent filterbase $\nabla$ in $X$. By use of the concept of $\theta$-regularity we easily establish a sufficient condition for $r$-subcontinuous functions to be subcontinuous.

LEMMA 4.1. An $r$-subcontinuous function into a $\theta$-regular space is subcontinuous.

The previous lemma along with the following result due to Fuller [10] will enable us to improve the result of Rose [1] that weakly continuous functions with closed graphs into rim-compact spaces are continuous.

LEMMA 4.2. A function with a closed graph is continuous if and only if it is subcontinuous.

THEOREM 4.3. An r-subcontinuous function with a closed graph into a $\theta$-regular space is continuous.

Since weakly continuous functions are $r$-subcontinuous, we have the following corollary.

COROLLARY 4.4. A weakly continuous function with a closed graph into a $\theta$-regular space is continuous.

The concept of C-continuous functions was introduced by Gentry and Hoyle [12]. Long and Hendrix [13] established that a function $\mathrm{f}: \mathrm{X} \rightarrow \mathrm{Y}$ is C-continuous if and only if $\mathrm{f}^{-1}(\mathrm{~B})$ is closed for each closed and compact subset $\mathrm{B}$ of $\mathrm{Y}$ and showed that every closed graph function is C-continuous. 
The proof of the following result which also improves Theorem 6 of (Rose [1]) is omitted since it is similiar to the proof of that theorem.

THEOREM 4.5. A weakly continuous $C$-continuous function into a rin-compact space is continuous.

It is shown by Rose [1] that every almost continuous function with a closed graph into a strongly locally compact space is continuous. We shall show that there is a class of functions containing both the class of weakly continuous functions and the class of almost continuous functions such that a function from this class into a strongly locally compact space is continuous if it is C-continuous.

DEFINITION 4.6. A function $\mathrm{f}: \mathrm{X} \rightarrow \mathrm{Y}$ is almost weakly continuous if $\mathrm{f}^{-1}(\mathrm{~V}) \subset$ $C \operatorname{Int}\left(\mathrm{Cl}\left(\mathrm{f}^{-1}(\mathrm{Cl}(\mathrm{V}))\right)\right)$ for each open subset $\mathrm{V}$ of $\mathrm{Y}$.

Clearly, weakly continuous and almost continuous functions are almost weakly continuous, but the separate converses are not true in general. Rose [7] presented an intersting comparison of almost continuity with continuity: A function $\mathrm{f}: \mathrm{X} \rightarrow \mathrm{Y}$ is (almost) continuous if and only if $\mathrm{f}(\mathrm{Cl}(\mathrm{U})) \subset \mathrm{Cl}(\mathrm{f}(\mathrm{I})$ ) for each (open) subset $\mathrm{U}$ of $\mathrm{X}$. The similiar result holds for almost weak continuity and weak continuity.

THEOREM 4.7. A function $\mathrm{f}: \mathrm{X} \rightarrow \mathrm{Y}$ is (almost) weakly continuous if and only if $f(C l(U)) \subset C 1_{\theta}(f(U))$ for each (open) subset $U$ of $X$.

PROOF. We prove the result only in the case of almost weak continuity. To prove

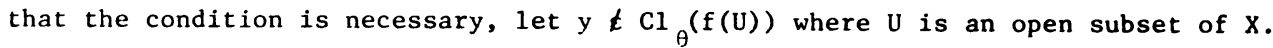
Then there exists an open subset $V$ containing y such that $c 1(V) \cap f(U)=\emptyset$. This implies that $\mathrm{f}^{-1}(\mathrm{Cl}(\mathrm{V})) \cap \mathrm{U}=\emptyset$ and consequent $1 \mathrm{y}$, Int $\left(\mathrm{Cl}\left(\mathrm{f}^{-1}(\mathrm{Cl}(\mathrm{V}))\right)\right) \cap \mathrm{Cl}(\mathrm{U})=\emptyset$. Since $\mathrm{f}$ is almost weakly continuous, $\mathrm{f}^{-1}(\mathrm{~V}) \cap \mathrm{Cl}(\mathrm{U})=\emptyset$. Therefore $\mathrm{V} \cap \mathrm{f}(\mathrm{Cl}(\mathrm{U}))=\emptyset$ and

$\mathrm{y} \notin \mathrm{f}(\mathrm{Cl}(\mathrm{U}))$. This shows that $\mathrm{f}(\mathrm{Cl}(\mathrm{U})) \subset \mathrm{Cl}_{\theta}(\mathrm{f}(\mathrm{U}))$.

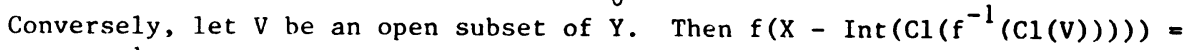
$=\mathrm{f}\left(\mathrm{Cl}\left(\operatorname{Int}\left(\mathrm{f}^{-1}(\mathrm{Y}-\mathrm{Cl}(\mathrm{V}))\right)\right)\right)$ and by hypothesis it follows that

$$
f\left(X-\operatorname{Int}\left(\mathrm{Cl}\left(\mathrm{f}^{-1}(\mathrm{C} 1(\mathrm{~V}))\right)\right)\right) \subset \mathrm{Cl}_{\theta}\left(\mathrm{f}\left(\operatorname{Int}\left(\mathrm{f}^{-1}(\mathrm{Y}-\mathrm{Cl}(\mathrm{V}))\right)\right)\right) \text {. }
$$

This implies that

$$
\mathrm{f}\left(\mathrm{X}-\operatorname{Int}\left(\mathrm{Cl}\left(\mathrm{f}^{-1}(\mathrm{Cl}(\mathrm{V}))\right)\right)\right) \subset \mathrm{Cl} 1_{\theta}(\mathrm{Y}-\mathrm{C} 1(\mathrm{~V})) \subset \mathrm{Y}-\mathrm{V} \text {. }
$$

Therefore $\left(X-\operatorname{Int}\left(\mathrm{Cl}\left(\mathrm{f}^{-1}(\mathrm{Cl}(\mathrm{V}))\right)\right)\right) \cap \mathrm{f}^{-1}(\mathrm{~V})=\emptyset$. This shows that $\mathrm{f}$ is almost weakly continuous.

We remark that by Theorem 4.7 it follows that almost weakly continuous functions into regular spaces are almost continuous.

THEOREM 4.8. If $\mathrm{f}: \mathrm{X} \rightarrow \mathrm{Y}$ is an almost weakly continuous $\mathrm{C}$-continuous function into a strongly locally compact space $Y$, then $f$ is continuous.

PROOF. Let $\mathrm{V}$ be a basic open set in $\mathrm{Y}$ with compact closure. Since $f$ is C-continuous, $\mathrm{f}^{-1}(\mathrm{Cl}(\mathrm{V}))$ is closed so that the almost weak continuity of $f$ implies that $\mathrm{f}^{-1}(\mathrm{~V}) \subset \operatorname{Int}\left(\mathrm{f}^{-1}(\mathrm{Cl}(\mathrm{V}))\right)$. By Theorem 2 in (Rose [1]), $\mathrm{f}$ is weakly continuous and by Theorem 4.5 , $\mathrm{f}$ is continuous.

COROLLARY 4.9. If $\mathrm{f}: \mathrm{X} \rightarrow \mathrm{Y}$ is an almost weakly continuous function with a closed graph into a strongly locally compact space $Y$, then $f$ is continuous. 


\section{REFERENCES}

1. Rose, D.A. On Levine's decomposition of continuity, Canad. Math. Bu11. 21 (1978), 477-481.

2. Veličko, N.V. H-closed topological spaces, Mat. Sb. 70 (112) (1966), 98-112 Amer. Math. Soc. Transl. 78 (2) (1968), $\frac{103-118 .}{103}$

3. Scarborough, C.T. and Stone, A.H. Products of nearly compact spaces, Trans. Amer. Math. Soc. 124 (1966), 131-147.

4. Steen, L.A. and Seebach, J.A. Counterexamples in Topology, Holt, Rinehart and Winston, Inc., New York, 1970 .

5. Levine, N. A decomposition of continuity in topological spaces, Amer. Math. Monthly $68(1961), 44-46$.

6. Husain, T. Almost continuous mappings, Prace Mat. 10 (1966), 1-7.

7. Rose, D.A. Weak continuity and almost continuity, preprint.

8. Davis, A.S. Indexed systems of neighborhoods for general topological spaces, Amer. Math. Month1y 68 (1961), 886-893.

9. Janković, D.S. On some separation axioms and $\theta$-closure, Mat. Vesnik 4 (17) $\underline{32}$ $(1980), 439-449$.

10. Fuller, R.V. Relations among continuous and various non-continuous functions, Pacific J. Math. 25 (1968), 495-509.

11. Herrington, L.L. Remarks on H(i) spaces and strongly-closed graphs, Proc. Amer. Math. Soc. 52 (1975), 417-426.

12. Gentry, K.R. and Hoyle, H.B. C-continuous functions, Yokohama Math. Journal 18 (1970), 71-76.

13. Long, P.E. and Hendrix, M.D. Properties of C-continuous functions, Yokohama Math. Journal $22(1974), 117-123$. 


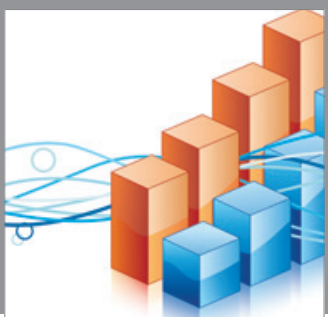

Advances in

Operations Research

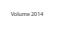

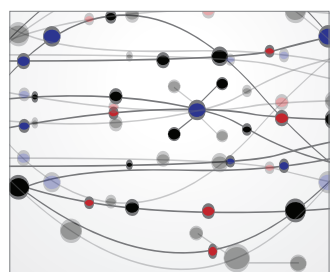

\section{The Scientific} World Journal
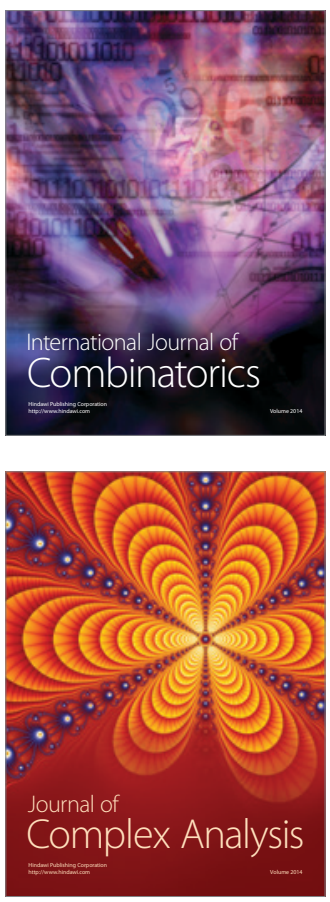

International Journal of

Mathematics and

Mathematical

Sciences
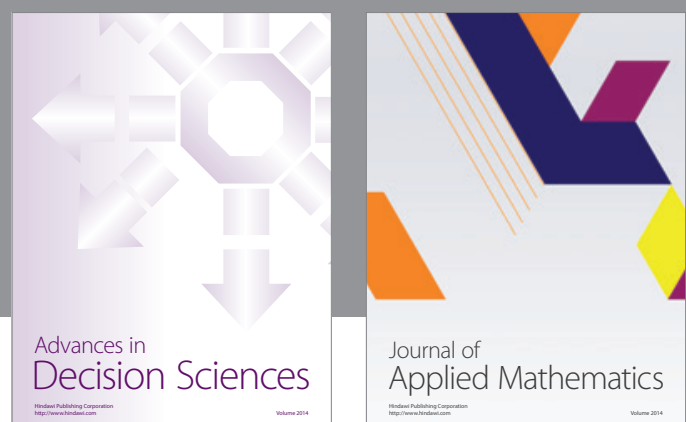

Journal of

Applied Mathematics
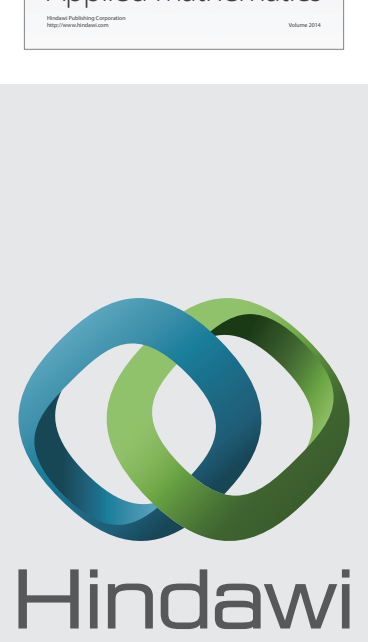

Submit your manuscripts at http://www.hindawi.com
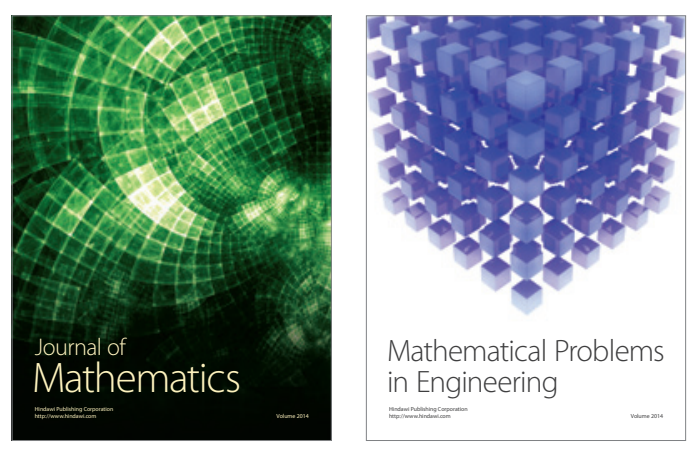

Mathematical Problems in Engineering
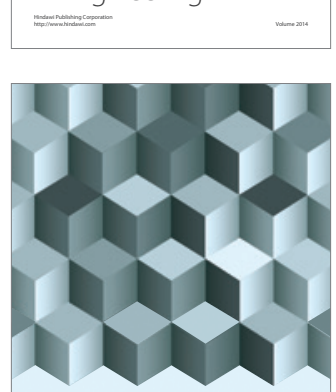

Journal of

Function Spaces
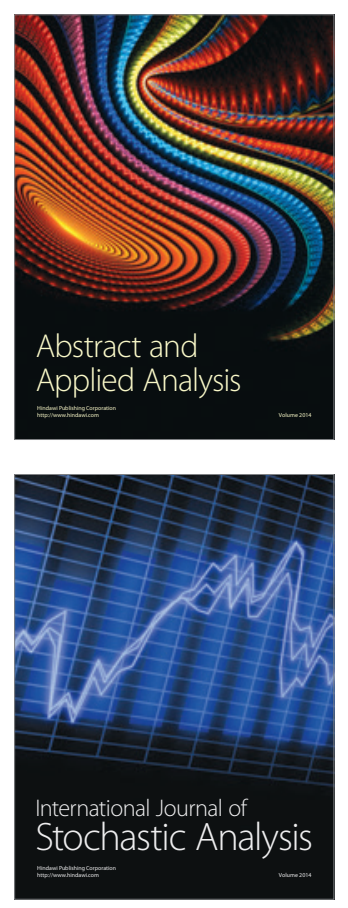

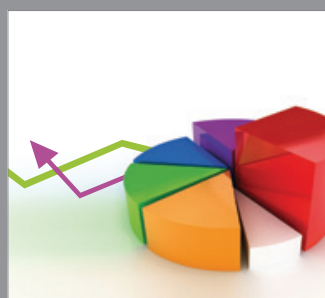

ournal of

Probability and Statistics

Promensencen
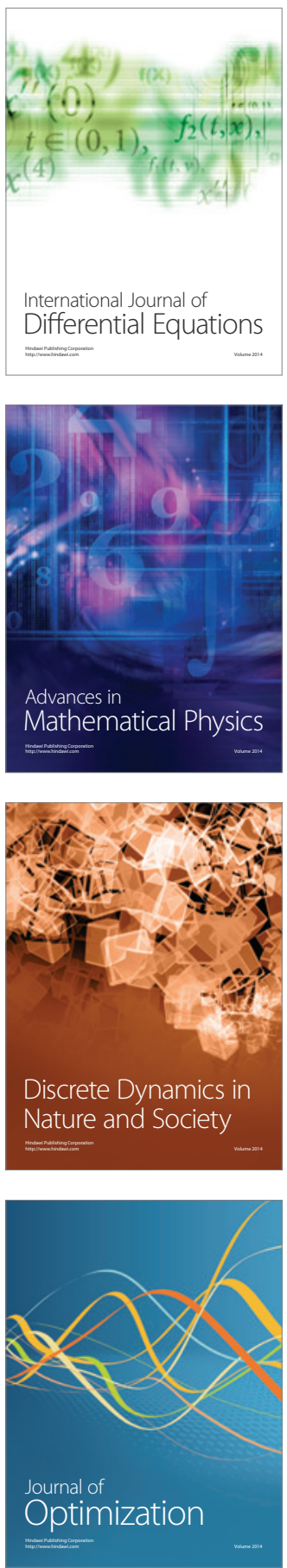